\title{
Metabolic profile, obesity and overweight among Sri Lankan males with ischaemic heart disease: A single centre experience
}

\author{
Wickramatilake $\mathbf{C M}^{1}$, Mohideen $\mathrm{MR}^{2}$, Pathirana $\mathrm{C}^{1}$ \\ ${ }^{I}$ Department of Biochemistry, Faculty of Medicine, University of Ruhuna, Galle, Sri Lanka. \\ ${ }^{2}$ International Medical University, IMU Clinical School, 6 Jalan Indah, Taman Sri Kenangan 83000, BatuPahat \\ Johor, Malaysia.
}

Correspondence: Dr. C.M. Wickramatilake e-mail: chandimadhu@live.com

iD https://orcid.org/0000-0003-0787-5144

\begin{abstract}
Introduction: Obesity is a significant risk factor for ischaemic heart disease (IHD). Asians who are not overweight or obese tend to have IHD and develop metabolic derangements in their early life. We investigated the presence of metabolic derangements and overweight and obesity in male patients with IHD in comparison to a control group.

Methods: Two hundred and six male patients with IHD at the Cardiothoracic Unit and the Coronary Care Unit and 103 male controls at the Surgical Units, Teaching Hospital Karapitiya were included. Demographic data, anthropometric measurements and blood samples were collected. World Health Organization (WHO) cut-off values defined for Asians were used to define obesity using body mass index (BMI) and waist circumference (WC). Plasma glucose, serum lipids and high-sensitivity C-Reactive protein (hs-CRP) were estimated. Group comparison was done using appropriate statistical tests. Controlling for age (adjustments) was done for anthropometric variables and biochemical variables before comparison.
\end{abstract}

Results: The proportions with overweight or generalised obesity defined by BMI were not significantly different in both patient groups \{patients with angiographically-proven coronary artery disease and first acute ST-elevation myocardial infarction (STEMI) $\}$ compared to controls (all $p>0.05$ ). However, the proportion of patients with central obesity was higher in patients with angiographically-proven coronary artery disease $(p=0.044)$, while a difference was not observed in patients with STEMI $(p=0.193)$. The mean concentration of plasma glucose, serum lipids and hs-CRP were significantly higher in patients compared to controls (all $p<$ $0.05)$.

Conclusions: Proportions of patients with overweight and generalised obesity were not significantly higher in both patient groups compared to controls. However, the proportion of patients with central obesity was significantly higher among patients with angiographically-proven coronary artery disease group compared to the controls.

Keywords: Anthropometry, ischaemic heart disease, males, metabolic profile, obesity, Sri Lanka 


\section{Introduction}

Obesity is defined as excess body fat and is a significant health problem which is known as a major risk factor for ischaemic heart disease (IHD) (1-2). Body mass index (BMI) is used to define the generalised obesity among the anthropometric parameters (3). Waist circumference measures central obesity that reflects visceral fat (4). World Health Organization (WHO) has recommended cutoff values for these parameters to define obesity and to identify increased cardiovascular risk (5). Asians who are not overweight or obese tend to have IHD and develop metabolic derangements such as dyslipidaemia, diabetes mellitus or prediabetes and chronic systemic inflammation in their early life (6). The availability of research data is limited to evaluate the usefuness of common anthropometric measurements to assess the overweight and obesity at the local set up. Therefore we wanted to compare the proportion of patients with obesity and metabolic derangements between two groups of patients with IHD and hospital-based controls who did not have clinically manifested coronary artery disease.

\section{Methods}

This was a hospital-based, case-control study which included 103 male patients with first clinically manifested acute ST-elevation myocardial infarction (STEMI) and 103 male patients with angiographically-proven coronary artery disease (CAD) awaiting coronary artery bypass grafting at Teaching Hospital, Karapitiya, Sri Lanka. The control group consisted of 103 male surgical patients awaiting minor surgeries with normal Electrocardiogramme at General Surgical Units, Teaching Hospital, Karapitiya. Patients and the controls in the age range of 30 to 70 years were recruited. Common exclusion criteria for three groups of participants were history of recent surgery or major trauma within three months or a history of acute coronary syndrome in the past three months, malignancy, chronic inflammatory disorders, current acute severe infections (C-reactive protein level more than $10 \mathrm{mg} / \mathrm{dL}$ ) and dementia or any structural damage to the central nervous system.
An interviewer-administered questionnaire was used to collect the relevant information from the study subjects. The principle investigator, a medically qualified doctor collected the data using the same instruments. Weight, height and waist circumference measurements were obtained according to the standard protocols (7). Serum lipids and plasma glucose were estimated based on spectrophotometry based techniques (ProDia International, UAE). High sensitivity-CRP was estimated using turbidimetry(CRP XL WIDE RANGE, DIAgAM, Rue du Parc Industriel, 7822, Ghislenghien, Belgium). Generalised obesity was defined using BMI according to the cut-off values $\left(\geq 25 \mathrm{~kg} / \mathrm{m}^{2}\right)$ given for Asians by WHO (8). Waist circumference of $94 \mathrm{~cm}$, set by WHO was used to define central obesity of males (9). Data were analysed using Minitab version 15 for Windows. Controlling for age (adjustments) was done for anthropometric variables and biochemical variables before comparison.

Two-proportion test and independent two sample t-test were used in the analysis. Significance level was defined as 0.05 . Ethical clearance was obtained from the Ethical Review Committee of Faculty of Medicine, University of Ruhuna, Sri Lanka. Informed written consent was obtained from all the participants before the data collection. Conduct of the research project was according to the Declaration of Helsinki. Confidentiality of data was strictly maintained.

\section{Results}

Table 1 shows a comparison of baseline characteristics of the two categories of patients and controls. The mean concentration of plasma glucose, serum lipids and high sensitivity $\mathrm{C}$-reactive protein (hs-CRP) were significantly higher in patients of both groups compared to controls (all $p<0.05$ ).

Table 2 shows the comparison of overweight, generalised obesity and central obesity among the two groups of patients and controls according to WHO defined cut-off levels for Asians. 
Table 1: Comparison of baseline characteristics of two patient categories vs. controls

\begin{tabular}{lccccc}
\hline Characteristics & $\begin{array}{c}\text { Controls } \\
\mathbf{n}=\mathbf{1 0 3}\end{array}$ & $\begin{array}{c}\text { Angiographically } \\
\text { proven CAD } \\
\mathbf{n = 1 0 3}\end{array}$ & $\begin{array}{c}\boldsymbol{p} \\
\text { value }\end{array}$ & $\begin{array}{c}\text { STEMI } \\
\text { patients } \\
\mathbf{n}=\mathbf{1 0 3}\end{array}$ & $\begin{array}{c}\boldsymbol{p} \\
\text { value }\end{array}$ \\
\hline Age (years) & $52 \pm 11$ & $57 \pm 8$ & 0.001 & $54 \pm 8$ & 0.201 \\
BMI (kgm $\left.{ }^{-2}\right)$ & $22.4 \pm 5$ & $23.9 \pm 3$ & $0.008^{*}$ & $21.2 \pm 3.6$ & $0.055 \dagger$ \\
Waist circumference (cm) & $75.9 \pm 10$ & $87.9 \pm 7$ & $0.001 *$ & $76.9 \pm 9.9$ & $0.462 \dagger$ \\
Regular physical activity & $15(14.6 \%)$ & $17(16.5 \%)$ & 0.267 & $14(13.6 \%)$ & 0.433 \\
hs-CRP (mg/L) & $1.7 \pm 0.6$ & $3.4 \pm 1.62$ & 0.001 & $3.7 \pm 0.84$ & 0.001 \\
TGs (mmol/L) & $1.5 \pm 0.8$ & $2.5 \pm 1.0$ & 0.001 & $2.1 \pm 1.0$ & 0.001 \\
TCh (mmol/L) & $5.2 \pm 1.6$ & $5.9 \pm 2.8$ & 0.022 & $6.0 \pm 2.4$ & 0.001 \\
HDL-Ch (mmol/L) & $1.4 \pm 0.6$ & $1.1 \pm 0.5$ & 0.001 & $1.1 \pm 0.5$ & 0.001 \\
LDL-Ch (mmol/L) & $3.1 \pm 0.5$ & $3.9 \pm 1.2$ & 0.001 & $4.5 \pm 2.4$ & 0.001 \\
PG (mmol/L) & $5.1 \pm 0.6$ & $5.5 \pm 1.4$ & 0.007 & $6.0 \pm 2.06$ & 0.001 \\
\hline
\end{tabular}

*Remained significantly different after the age-adjustments. $\dagger$ Become significantly different after age-adjustment. Comparison was made between the two patient groups and the control group separately. Two-proportion test and Independent sample $t$-test were used in the analysis. Data presented as frequencies, percentages and mean $\pm \mathrm{SD} . \mathrm{CAD}=$ Coronary artery disease, $\mathrm{BMI}=$ Body mass index. STEMI $=$ ST- elevation myocardial infarction. hs-CRP $=$ High sensitivity-C-reactive protein, TGs $=$ Triglycerides, TCh $=$ Total cholesterol, HDL-Ch $=$ High density lipoprotein cholesterol, $\mathrm{LDL}-\mathrm{Ch}=$ Low density lipoprotein cholesterol, $\mathrm{PG}=$ Plasma glucose .

Table 2: Comparison of overweight, generalised obesity and central obesity among patients and controls according to WHO defined Asian cut-off levels

\begin{tabular}{|c|c|c|c|c|c|c|}
\hline Category & & $\begin{array}{l}\text { Controls } \\
n=103)\end{array}$ & $\begin{array}{c}\text { Angiographically- } \\
\text { proven } \\
\text { CAD patients } \\
(\mathbf{n}=103)\end{array}$ & p value & $\begin{array}{l}\text { STEMI } \\
\text { patients } \\
(n=103)\end{array}$ & p value \\
\hline \multicolumn{7}{|c|}{ Body mass index $\left(\mathrm{kg} / \mathrm{m}^{2}\right)$} \\
\hline$<18.5-$ underweight & 17 & $(16.5 \%)$ & $0 \quad(0.0 \%)$ & 0.001 & $26(25.2 \%)$ & 0.121 \\
\hline 18.5 - 22.9 - normal & 45 & $(43.7 \%)$ & $43 \quad(41.7 \%)$ & 0.778 & $52(50.5 \%)$ & 0.327 \\
\hline 23 - 24.9 - overweight & 20 & $(19.4 \%)$ & $31 \quad(30.1 \%)$ & 0.074 & $13(12.6 \%)$ & 0.182 \\
\hline$\geq 25$ - obesity & 21 & $(20.4 \%)$ & $29 \quad(28.2 \%)$ & 0.192 & $12(11.7 \%)$ & 0.085 \\
\hline \multicolumn{7}{|c|}{ Waist circumference (cm) } \\
\hline$<94$ & 96 & $(93.2 \%)$ & $87(84.5 \%)$ & 0.044 & $100(97.1 \%)$ & 0.193 \\
\hline$\geq 94$ & 7 & $(6.8 \%)$ & $16(15.5 \%)$ & 0.044 & $3(2.9 \%)$ & 0.193 \\
\hline
\end{tabular}

Data presented as frequencies and percentages. $\mathrm{CAD}=$ Coronary artery disease, $\mathrm{BMI}=$ Body mass index. $\mathrm{STEMI}=\mathrm{ST}$-elevation myocardial. Comparison was made between the two patient groups and the control group separately. Two-proportion test was used in the analysis.

The proportions with overweight or obesity were not significantly different in both patient groups \{patients with angiographically-proven coronary artery disease and patients with first acute STelevation myocardial infarction (STEMI) $\}$ defined by BMI compared to controls (all $\mathrm{p}>0.05$ ).
However, the proportion of patients with central obesity was higher in patients with angiographicallyproven coronary artery disease $(p=0.044)$, while a difference was not observed in patients with $\operatorname{STEMI}(p=0.193)$. 


\section{Discussion}

According to the findings of our study, in spite of the presence of coronary artery disease and related metabolic derangements, the proportion of patients with generalised obesity assessed by BMI is not significantly higher in the patient groups compared to controls. However, the percentage of patients with central obesity assessed by waist circumference among those with angiographically-proven coronary artery disease showed a significant difference. Interestingly, patients in the underweight category had developed STEMI. Although obesity and overweight were not highly prevalent in both disease groups, there were biochemically identified metabolic changes among the patients.

The relationship and the behavior of anthropometric parameters, body compositions and body fat are different in various ethnic groups across the world population. Asians generally have high fat content for a given BMI and hence, they have an increased risk of CAD and metabolic disease (10). Therefore, it is debatable whether the defined cut-off values of these anthropometric parameters accurately reflect the amount of body fat or visceral fat. In certain instances BMI is known to overestimate the degree of obesity in people with lower body fat percentage and higher lean body mass (11) or sometimes underestimate and reflect as low body fat especially in Asians (6). If WHO defined cut-off values for Europids are used for Asians (5) the detection rate of overweight and obesity could be further lowered.

A recent study done on Koreans revealed that the individuals with normal weight obesity (normal BMI with high body fat by body composition assessment) had subclinical atherosclerosis (12). The probable explanation for the observation is that atherosclerosis and related metabolic alterations are mainly driven by visceral fat which is not reflected by BMI. A study done on Indians showed that Asian Indians have excess cardiovascular risk at normal BMI and WC values. It suggests that definitions of "normal" ranges of BMI and WC need to be revised for Asian Indians (13). In spite of normal BMI, Asians tend to have impaired cardiovascular metabolic profile which is associated with subclinical vascular inflammation (14).
There are few local studies done to assess the validity of the current cut-off values of the BMI and WC to define the obesity. One study revealed that BMI and WC values of $24 \mathrm{~kg} / \mathrm{m}^{2}$ and $92 \mathrm{~cm}$ can be considered appropriate cut-off values when detecting central obesity in Sri Lankan premenopausal women (15). Another study suggested that mean BMI and WC for Sri Lankan males as $21.1 \mathrm{kgm}^{-2}$ and $78 \mathrm{~cm}$ and they further commented that Sri Lankans have a higher prevalence of obesity which may not be detected by the WHO defined guidelines for Asians $(8,16)$. Moreover, they found that the percentage of Sri Lankan adults in the overweight, obese and centrally obese categories were $25.2 \%, 9.2 \%$ and $26.2 \%$ respectively (16). A multi-national study demonstrated that there are possibilities of frequent discordances between BMI, WC and visceral fat mass among different ethnic and racial groups (4). However, Sri Lanka experiences double burden of nutritional disorders and further lowering the cut-off value of BMI to define the obesity is dubious, because detection of underweight and undernutrition may get influenced. Therefore, the incorporation of the metabolic markers with obesity in the assessment of cardiometabolic risk could be an option.

Dyslipidaemia, elevated blood glucose and inflammation are known to increase the risk of atherosclerosis and IHD (17). Metabolic alterations that occurs in the individuals with normal body anthropometry is said to be due to the increased fat content in the body especially the visceral fat (14). Both patient groups showed significant metabolic alterations in the present study. The prevalence of diabetes mellitus in Sri Lanka was nearly $11 \%$, while $1 / 5^{\text {th }}$ of the adult population was suffering from dysglycaemia $(18,19)$. A recent local study has found that metabolic syndrome as a common health concern among Sri Lankan adults and prevalence of metabolic syndrome in adult males was $18.4 \%(20)$.

\section{Conclusions}

Compared to controls, the proportions of patients with overweight and generalized obesity in both patient groups with ischaemic heart disease were not significantly higher, though they had related metabolic derangements. However, the proportion 
of patients with central obesity was significantly higher among patients with angiographically-proven coronary artery disease group compared to the control group. This could be due to the fact that Sri Lankans may develop ischaemic heart disease even without obesity since they are metabolically impaired or existing obesity is underestimated by the cut-off levels used.

\section{Recommendations}

We wish to recommend larger multi-center studies which should include body composition assessment.

\section{Limitations}

Use of hospital based control group is a limitation of this study.

\section{Acknowledgements}

We wish to acknowledge the University Grants Commission, Sri Lanka (UGC/ICD/CRF/2009/2/ 47) and Faculty Grant for funding the project. We sincerely thank the staff at the Cardiothoracic Unit, Coronary Care Unit and the General Surgical Units at Teaching Hospital Karapitiya, staff at the Department of Biochemistry, Faculty of Medicine, University of Ruhuna.

\section{Conflict of Interest}

None.

\section{References}

1. Flegal KM, Carroll MD, Kit BK, Ogden CL. Prevalence of obesity and trends in the distribution of body mass index among US adults, 1999-2010. JAMA, 2012; 307(5): 491-497.

2. Kim MK, Lee WY, Kang JH, Kang JH, Kim BT, Kim SM, et al. 2014 clinical practice guidelines for overweight and obesity in Korea. Endocrinol Metab, 2014; 29(4): 405-409.

3. Weisell RC. Body mass index as an indicator of obesity. Asia Pac JClin Nutr, 2002; 11(Supp1 8): S681-684.

4. Nazare JA, Smith J, Borel AL, Aschner P, Barter P, Van Gaal L, et al. Usefulness of measuring both body mass index and waist circumference for the estimation of visceral adiposity and related cardiometabolic risk profile (from the INSPIRE ME IAA study). Am J Cardiol, 2015; 115(3): 307-15.

5. World health organization, 1998. Obesity: Preventing and Managing the Global Epidemic. Geneva, WHO. Available at: http://whqlibdoc.who.int/hq/1998/WHO_NUT_ NCD_98.

6. Low S, Chin MC, Ma S, Heng D, Deurenberg-Yap M. Rationale for redefining obesity in Asians. Ann Acad Med Singapore, 2009; 38(1): 66-69.

7. National Health and Nutrition Examination Survey III. Body Measurements (Anthropometry) Westat, Inc. 1650 Research Boulevard Rockville, MD 20850(301); 2511500 .

8. International Association for the Study of Obesity (IASO) and International Obesity Task Force (IOTF), 2000. The Asia - Pacific Perspective; Redefining obesity and its treatment, WHO Western Pacific Region 2000.

9. WHO Report of Expert Consultation on waist circumference and waist-to-hip ratio. Geneva, 2008.

10. Wen CP, David Cheng TY, Tsai SP, Chan HT, Hsu HL, Hsu CC, et al. Are Asians at greater mortality risks for being overweight than Caucasians? Redefining obesity for Asians. Public Health Nutr, 2009; 12(4): 497-506.

11. Romero-Corral A, Somers VK, Sierra-Johnson J, Thomas RJ, Collazo-Clavell ML, Korinek J, et al. Accuracy of body mass index in diagnosing obesity in the adult general population. Int JObes (Lond), 2008; 32(6): 959-966.

12. Kim S, Kyung C, Park JS, Lee SP, Kim HK, Ahn CW, et al. Normal-weight obesity is associated with increased risk of subclinical atherosclerosis. Cardiovasc Diabetol, 2015; 14: 58 .

13. Vikram NK, Pandey RM, Misra A, Sharma R, Devi JR, Khanna N. Non-obese (body mass index $<25 \mathrm{~kg} / \mathrm{m}^{2}$ ). Asian Indians with normal waist circumference have high cardiovascular risk. Nutrition, 2003; 19(6): 503-9.

14. Kang S, Kyung C, Park JS, Kim S, Lee SP, Kim MK, et al. Subclinical vascular inflammation in subjects with normal weight obesity and its association with body fat: an 18F-FDG-PET/CT study. Cardiovasc Diabetol, 2014; 13: 70 .

15. Nanayakkara J, Lekamwasam S. Validity of BMI, hip and waist circumferences as surrogate measures of obesity in a cohort of Sri Lankan premenopausal women. Ceylon Med J, 2013; 58(2): 72-5. 
16. Katulanda P, Jayawardena MA, Sheriff MH, Constantine GR, Matthews DR. Prevalence of overweight and obesity in Sri Lankan adults. Obes Rev, 2010; 11(11): 751-6.

17. Goswami B, Tayal D, Tyagi S, Mallika V. Assessment of insulin resistance, dyslipidaemia and inflammatory response in North Indian male patients with angiographically proven coronary artery disease. Minerva Cardioangiologica, 2011;59(2): 139-147.

18. Katulanda P, Constantine GR, Mahesh JG, Sheriff R, Seneviratne RDA, Wijeratne $\mathrm{S}$, et al. Prevalence and projections of diabetes and pre-diabetes in adults in Sri Lanka-Sri Lanka Diabetes. Cardiovascular Study (SLDCS) Diabet Med, 2008; 25: 1062-1069.
19. Wijewardene K, Mohideen MR, Mendis S, Fernando DS, Kulathilaka T, Weerasekara D, et al. Prevalence of hypertension, diabetes and obesity: baseline findings of a population based survey in four provinces in Sri Lanka. Ceylon Med J, 2005; 50: 62-70.

20. Katulanda P, Ranasinghe P, Jayawardana R, Sheriff R, Matthews DR. Metabolic syndrome among Sri Lankan adults: prevalence, patterns and correlates. Diabetol Metab Syndr, 2012; 4(1): 24 